\title{
Author's name
}

Michele Luchetti

\author{
Affiliation \\ Department of Philosophy, University of Geneva
}

\section{Title}

From successful measurement to the birth of a law: disentangling coordination in Ohm's scientific practice

\section{Keywords}

Measurement

Coordination

$\mathrm{Ohm}$

Scientific practice

Calibration

\section{Abstract}

In this paper, I argue for a distinction between two scales of coordination in scientific inquiry, through which I reassess Georg Simon Ohm's work on conductivity and resistance. Firstly, I propose to distinguish between measurement coordination, which refers to the specific problem of how to justify the attribution of values to a quantity by using a certain measurement procedure, and general coordination, which refers to the broader issue of justifying the representation of an empirical regularity by means of abstract mathematical tools. Secondly, I argue that the development of Ohm's measurement practice between the first and the second experimental phase of his work involved the change of the measurement coordination on which he relied to express his empirical results. By showing how Ohm relied on different calibration assumptions and practices across the two phases, I demonstrate that the concurrent change of both Ohm's experimental apparatus and the variable that Ohm measured should be viewed based on the different form of measurement coordination. Finally, I argue that Ohm's assumption that tension is equally distributed in the circuit is best understood as part of the general coordination between Ohm's law and the empirical regularity that it expresses, rather than measurement coordination. 


\section{Introduction}

In the last two decades, several studies in philosophy of science have focused on measurement in relation to other epistemic activities. These works have been considered as an emerging research programme lacking continuity with classic $20^{\text {th }}$ century formal approaches to measurement (Tal 2013). ${ }^{1}$ One concern central to this scholarship is how scientists justify their belief that certain measurement procedures identify the quantity of interest in the absence of independent methods to assess them. In recent literature, this has been described as the 'problem of nomic measurement' or the issue of 'coordination' between quantities and measurement procedures. ${ }^{2}$ In order to solve this problem, Chang (2004) and van Fraassen (2008) suggest considering the meaning of quantity concepts as emerging along the process through which a form of coordination with measurement procedures is achieved, thus emphasising the constructive aspect of the dynamics between theorising and measuring. Their work stimulated the production of a wealth of case studies focusing on this issue across scientific disciplines (e.g., Barwich \& Chang 2015, McClimans et al. 2017, Michel 2019, Ruthenberg \& Chang 2017, Tal 2016a). Further contributions have significantly advanced the study of coordination with respect to measurement. These works provided in-depth analyses of epistemic components internal to the measurement process, for example, calibration, and clarified crucial epistemological distinctions such as the one between instrument readings and measurement outcomes (e.g., Boumans 2007, Frigerio et al. 2010, Giordani \& Mari 2012, Tal 2016b, 2017b, 2017c).

However, despite its outstanding achievements, this literature rarely acknowledges or investigates the relationship between the specific issue of coordination in measurement and the more general problem of coordination between theory and phenomena. The issue of coordinating quantities with measurement procedures may, in fact, be viewed as a special case of the more general issue of providing the conditions of applicability of theoretical terms to concrete phenomena. Classic analyses of this general issue of coordination were mainly developed from a conventionalist standpoint within the logical empiricist tradition. ${ }^{3}$ In turn, the general issue of coordination between abstract theoretical terms and concrete phenomena directly relates to the problem of justifying the use of mathematically expressed laws to represent empirical regularities (Reichenbach 1920, Friedman 2001, 2009).

\footnotetext{
${ }^{1}$ Representative works in this tradition include Campbell (1920), Stevens (1946), and Suppes (1951).

2 Chang (2004) labels this issue the 'problem of nomic measurement', followed by other scholars (e.g., Boumans 2005, Cartwright \& Bradburn 2011, Sherry 2011). However, in the SEP page on 'Measurement in science', Tal (2017a: section 8.1) points out that "this circularity has been variously called the "problem of coordination" (van Fraassen 2008: ch. 5) and the "problem of nomic measurement" (Chang 2004: ch. 2)". This statement mirrors the rather interchangeable use of both expressions in the literature. Since my goal in this paper is not one of semantic cleaning, I will not discuss the legitimacy of the use of these expressions.

${ }^{3}$ Mach (1896) is usually considered an antecedent.
} 
The main goal of this paper is to outline the distinction between these two kinds of coordination and apply it to the inquiry into electrical conductivity conducted by Georg Simon Ohm [1789-1854]. Ohm was the German physicist and mathematician who famously discovered the law expressing the direct proportionality between voltage and current intensity and the inverse proportionality between current intensity and resistance. Despite the continued widespread use of his law, Ohm's own scientific endeavours have rarely been considered in detail by philosophers, ${ }^{4}$ and historical interest in Ohm has been sporadic in relation to his historical and contemporary epistemological importance. ${ }^{5}$ I will show that Ohm's scientific practice offers an excellent example of how understanding the relationship between these two kinds of coordination that operate on different scales can be fruitful for epistemological analyses, even though this distinction has been overlooked by the existing literature.

In section 2, I will first introduce the issue of coordination in the epistemology of measurement by briefly presenting Chang's (2004) approach. He and van Fraassen (2008) developed the two most influential recent accounts tackling this epistemological issue. However, for the purposes of this paper, I will consider their differences to be negligible, and Chang's view provides a more suitable background, due to its tighter integration with a historical case study. Second, I will introduce my conceptual distinction between two scales of coordination, one related to the narrower problem of how to justify measuring a quantity by means of a certain measurement procedure, the other referring to the broader issue of justifying the representation of an empirical regularity by means of abstract mathematical tools. Finally, I will present some categories that have been recently discussed by epistemologists of measurement, which will enable a rich and fruitful analysis of the internal components of measurement. These categories will be useful for reconstructing, in section 3, two different phases of Ohm's experimental practice. By analysing the details of his measurement procedures and, more precisely, those concerning calibration, I will argue that the narrower kind of coordination - on which Ohm relied to express his empirical results - changed from the first to the second experimental phase, where the latter is the one in which he obtained his famous law. Then, I will show that this narrower kind of coordination, although crucial to Ohm's inquiry, was not the only one involved in the achievement of his law. By analysing his 1827 mathematical treatise, I will argue that a theoretical assumption - that tension is equally distributed between every two points of a circuit - provided part of the justification for the more general form of

\footnotetext{
${ }^{4}$ A notable exception is the logical reconstruction provided by Heidelberger (1980).

${ }^{5}$ Many historical efforts have focused on understanding the reasons of the late reception of Ohm's work by the scientific community. Among the possible causes of Ohm's late reception, historians have identified the widespread impression that Ohm's law resulted from a purely theoretical deduction (Shedd \& Hershey 1913), the hostility of the dominant Hegelian philosophy towards rigorous empirical researches (Winter 1944), his unorthodox use of the notion of 'tension' (Schagrin 1963), and the highly mathematical character of his treatment, compared to the standards of contemporary German science (Caneva 1978).
} 
coordination between Ohm's mathematical formulation of his law and the empirical regularity that it expresses. In section 4, I will summarise my conclusions.

\section{Circularity, measurement, and coordination}

\subsection{The problem of circularity in measurement and Chang's solution}

Measuring practices have a pervasive role in establishing evidence and providing certain specific conditions for the applicability of abstract representations to phenomena. The relationship between abstract terms expressing quantities and the ways of measuring those quantities is key to understanding the problem of circularity discussed in the literature as the 'problem of nomic measurement', or the issue of 'coordination' between quantities and measurement procedures.

To measure a quantity, we often infer its value from the values of other quantities, as when we 'read' the temperature of a room from the length of the mercury column in a thermometer hanging on the wall. This inference is based on the knowledge of the physical law that describes the relationship between the quantities of temperature and length in a specific physical interaction. Such a 'measurement law' represents the measurement interaction through which the desired quantity is inferred from other quantities (Chang 1995). Yet how do we know the form of the function that relates the values of temperature and length? This question leads to a conundrum: we need to know the law to assess the soundness of a measurement procedure. However, it is usually through measurements that we establish and test empirical regularities. Clearly, if a certain measurement procedure has been used to establish the measurement law, its precision and accuracy cannot be assessed by means of the very same measurement law. Nonetheless, such cases are not infrequent in the history of science, in which it seems that "an understanding of measurement and what is measured is presupposed rather than established in our effort to assign meaningful values to the items in a scale" (McClimans 2013: 530). Put into a question, what is the justification for believing that the measurement procedures we are deploying in fact measure the quantity of interest if we lack independent means of assessing these procedures? Solving this problem of circularity equates to finding appropriate and independent sources of justification for a measurement procedure that assigns certain values to a quantity.

Chang (2004) focuses on the history of thermometry to develop his view of scientific progress and he treats the problem of circularity that I described above as a central issue related to it. According to Chang, the meaning of the quantity concept 'temperature' emerged from a process of cyclic feedback between theoretical advances and improvements in measurement standards. At various stages of scientific progress, the same term - 'temperature' - has been used with reference to different standards of observability and measurement, and it can assume different sets of values depending on the form of the 
relative measurement scale. ${ }^{6}$ In his historical narrative Chang concludes that scientific progress follows cycles of 'epistemic iteration', that is, "a process in which successive stages of knowledge, each building on the preceding one, are created in order to enhance the achievement of epistemic goals" (Chang 2004: 45). Chang emphasises the historical character of this enterprise, based on the mutual refinement of theoretical background and measurement procedures. Thus, improvements at the level of measuring techniques contribute, on the one hand, to the better operationalisation of the quantity term, which, in turn, enables the gathering of experimental data to support further theorising. On the other hand, theoretical advances allow for the refinement of the definition of the quantity, by providing a guide to experimental measuring practices. This process embodies a kind of virtuous circle guided by the search for coherence between the various methodological and theoretical assumptions. According to Chang, the improvement of measurement standards very often takes place quite independently of changes in theory, at least until theoretical advances are such that they can subsume and justify measurement procedures. Such a process lasted more than a century in the case of temperature and it may be viewed as ongoing for several other quantities (Riordan 2015).

\subsection{Two meanings of 'coordination'}

One consequence of Chang's framework is that a quantity cannot be assigned any value independently of both a measurement procedure that identifies its possible values and a form of coordination of the procedure with the rest of the conceptual apparatus in which the quantity concept is embedded. The solution to the problem of circularity in measurement - or the "problem of nomic measurement" in his terminology - equates to progressively refining the provisional forms of justification of the measurement procedure developed alongside the theoretical knowledge and experimental techniques available at a certain stage of scientific development. Advancements in the precision and reliability of measurement procedures emerge via a process of mutual refinement with theoretical approaches. Even among metrologists, the consensus seems to lie on this historical and coherentist understanding of how coordination between quantity concepts and their standards of measurement is achieved (Johansson 2014). It is in this sense that both Chang and van Fraassen (2008) claim that the meaning of quantity terms is never a 'given', but rather it emerges along the various stages of this process.

This sense of 'coordination', as the achievement of a stable justification for the identification of a quantity by means of a procedure for measuring it, does not exhaust the meaning of this notion as it has been used in the literature. Solving the issue of circularity in measurement is only one way in which a form of coordination between abstract theoretical concepts and concrete phenomena can be established. Indeed, some theoretical concepts do not refer to measurable quantities. Most importantly, justification for the

\footnotetext{
${ }^{6}$ Here I refer to Stevens' (1946) standard fourfold classification of measurement scales: nominal, ordinal, interval, ratio.
} 
referential relationship between theory and phenomena does not necessarily involve the epistemic dimension of measurement. For instance, this can be obtained by means of theoretical justification, by relying on certain general assumptions about reality, or through any combination of the above. In the literature, the distinction and the relationship between the specific issue of coordination in measurement and the more general problem of coordination between theory and phenomena are hardly acknowledged. Two exceptions are van Fraassen (2008), who assumes that these issues are related but does not further examine their relationship, and Padovani $(2015,2017)$, who aims to bridge the discussion on coordination in measurement with one on coordination between theory and phenomena by focusing on Reichenbach's philosophical heritage. However, an in-depth analysis of the distinction between these two scales of coordination is still lacking.

For this reason, in this part I introduce a distinction between two meanings of 'coordination'. As I will show with my historical case study in section 3, this distinction can be helpful for the analysis of specific points of scientific inquiry in which the two scales of coordination are entangled. 'Measurement coordination', here refers to both the process by which a solution to the issue of circularity in measurement is achieved and the condition, resulting from this process, by which certain measurement standards reliably contribute to the identification of a quantity within a certain scientific framework. 'General coordination' in turn refers to the broader process of coordinating abstract theoretical representations with concrete phenomena, independent of the specific issue of circularity in measurement. This understanding of 'coordination' bears directly on the issue of how theories in general can refer to empirical phenomena. For instance, a mathematically formulated law can express an empirical regularity only if its variables are properly coordinated with concrete phenomena, that is, if the referential relationships between the variables and the respective phenomena are justified. For this reason, I use 'general coordination' to indicate the condition, resulting from this process, by which an abstract, mathematically formulated law can justifiably represent an empirical regularity.

Achieving an improved form of measurement coordination involves several epistemic components (instruments, methodological, theoretical, and metaphysical assumptions, etc.). The activity of measuring is a complex one and it is entangled with experimentation, model-building, and theorising (Chang 2004: ch. 3, Tal 2017b). For this reason, examining the details of a measurement procedure can be essential to understanding measurement coordination and its relationship with general coordination. By 'details' I mean the different epistemic components of a measurement procedure that assume a particular justificatory role within a certain form of measurement coordination. These components can be specifically theoretical, pragmatic, or metaphysical assumptions involved in the modelling of the measurement procedure itself, parts of the material experimental apparatus, or further background theoretical commitments, etc. Indeed, Chang's work on temperature has provided a paradigmatic term 
of comparison with respect to its awareness of the importance of details in understanding how scientists justify conceptual and metrological advances. Equally important, van Fraassen (2008) stresses the central role of an epistemological distinction - between instrument readings and measurement outcomes - in the analysis of measurement coordination. Recent literature has also introduced an array of analytic concepts that facilitate the task of identifying the epistemic components that contribute to the justification for a certain form of coordination between quantities and the procedures by which to measure them.

In the next section, I introduce some metrological categories recently analysed by the growing literature on the epistemology of measurement, which extend specific aspects that van Fraassen and Chang's accounts deal with to a lesser extent, given their broader scope. Most importantly, these recent contributions clarify the distinction between instrument readings and measurement outcomes as well as the role of calibration in measurement coordination, which will be crucial to my analysis of Ohm's scientific inquiry.

\subsection{When details matter: disentangling measurement coordination}

Measurement procedures are physical interactions between one or more epistemic subjects, a material apparatus, and a phenomenon occurring in an environment. At the same time, the epistemic subjects purport to represent a certain relationship between quantities by means of the physical process taking place during the measurement interaction. In this sense, we can distinguish 'measurement' senso strictu, understood as the set of physical procedures and material instruments used for enacting a measurement procedure and, in some cases, (re)producing a phenomenon, from measurement in the broader sense, inclusive of its representational character and, therefore, of the host of inferential assumptions involved in its representational use. The difference between instrument readings and measurement outcomes is central to this distinction. ${ }^{7}$

Instrument readings are observations of the states of the material instrument used to provide a quantitative representation of a certain phenomenon, once the physical process enacted during the measurement procedure has arrived at its end-state. For instance, when we place a mercury thermometer under our armpit, we must wait for a certain amount of time until the mercury in the thermometer column has expanded according to our body temperature. The end-state of the physical process, in this case, is when the mercury stops expanding, whilst the instrument reading is the length reached by the mercury column.

\footnotetext{
${ }^{7}$ Cf. Tal (2017b) especially pp. 235-236 for a very clear exposition. As I have mentioned above, the importance of this conceptual difference for the understanding of measurement coordination was already stressed by van Fraassen (2008). Cf. also Giordani \& Mari (2012), and Tal (2013).
} 
In understanding how to construct and successfully perform a physical measurement procedure, the procedure itself is subject to calibration. Calibration is the process through which models of the measurement procedure are constructed and tested, by modelling uncertainties and systematic errors of the procedure (or across procedures measuring the same quantity) under idealised statistical and theoretical assumptions (Boumans 2007, Frigerio et al. 2010, Mari 2003, Tal 2017c). The aim of calibration is (ideally) to account for all possible sources of measurement error given the best standards of precision available and, therefore, to improve the accuracy of a measurement procedure. ${ }^{8}$ The outcome of the calibration process, that is, an explicit model of measurement or a less integrated set of assumptions, enables inferences from instrument readings to measurement outcomes, thus playing a crucial role in the coordination of quantity terms with empirical content (McClimans et al. 2017, Tal 2019). However, it is rare that calibration entirely precedes the performing of measurement, since the two are themselves subject to cycles of mutual refinement.

Based on the above considerations, it follows that measurement outcomes are, in part, the product of a modelling process, i.e., calibration, which has as its object a certain measurement procedure. Thus, measurement outcomes are "the best predictors of the observed end-states of a measurement process relative to a particular theoretical and statistical model of that process" (Tal 2016b: 5). By modelling possible measurement errors and other confounding factors, and through recourse to statistical and theoretical assumptions, measurement outcomes are inferred from certain instrument readings. This means that some of the content of measurement outcomes is imposed by adjusting inconsistent observations based on idealised background assumptions. When measuring temperature with a mercury thermometer, an outcome of $37.5^{\circ} \mathrm{C}$ is not simply the result of observing the instrument reading (i.e., the length of the mercury column). Inferring this outcome from the instrument reading presupposes an already constructed measurement scale, the identification of a function relating the measured quantity and the quantity through which the measured quantity is represented, the modelling of the possible measurement errors, and the reduction of the statistical relevance of confounding factors, etc.

It should be clear by now that the process of achieving a form of measurement coordination, through which quantity terms acquire meaning, is influenced by several theoretical assumptions with different roles at different epistemic stages. Importantly, the theoretical background available to the scientists performing measurement and calibration often provides them with measurement laws that are crucial to anchoring the calibration of a measurement procedure and, therefore, enabling inferences from

\footnotetext{
${ }^{8}$ As Tal (2017c: 34) points out, the term 'calibration' is often used with reference to "the empirical activity of detecting correlations among the indications of instruments, or between the indications of an instrument and a set of reference systems that are associated with fixed values. Values are then assigned to the indications of the instrument being calibrated so as to match previously known values, often along with a rule for extrapolating between (and beyond) those known values". This understanding mainly accounts for the theoretical practice of instrument making, which is only one aspect of calibration.
} 
instrument readings to produce measurement outcomes. However, theoretical background also takes the form of idealising statistical assumptions, which provide justification for the construction of measurement scales, that is, mathematical structures that enable the representation of measurement outcomes according to certain ordering, difference, and ratio relations. ${ }^{9}$ However, the multiple roles of theoretical background should not lead us to underestimate the centrality of pragmatic considerations, empirical testing, and material instrumentation during calibration, especially in those epistemic contexts in which a sound theoretical understanding of the measurement interaction is lacking.

In the next section, I will use the notions of material instruments, physical procedures, instrument readings, measurement outcomes, and calibration to analyse Ohm's experimental work on electric conductivity. These categories will provide a helpful guide to disentangle the measurement coordination between the quantities of 'loss of force' and 'exciting force', and the measurement procedures deployed by Ohm. More specifically, I will show how analysing the details of the calibration process is necessary to pinpoint which assumptions were crucial to the measurement coordination that $\mathrm{Ohm}$ relied on. This, in turn, will allow us to understand the role of measurement coordination with respect to the general coordination between Ohm's famous law and the empirical regularity that it captures.

\section{Measurement and coordination in Ohm's scientific inquiry}

In this section, I present a case study that provides support for my claim that there are two different kinds of coordination operating at different scales. While measurement coordination is related to the specific problem of how to justify the attribution of values to a quantity by means of a certain measurement procedure, general coordination refers to the broader issue of justifying the representation of an empirical regularity by means of abstract mathematical tools. By analysing the details of Ohm's measurement procedures and, more precisely, those concerning calibration, I will argue that the measurement coordination on which he relied to express his empirical results changed in the transition from the first experimental phase to the second. Then, I will show that, even though the measurement coordination on which $\mathrm{Ohm}$ relied in the second phase was crucial to expressing the empirical results from which he derived his law, it was not sufficient for the general coordination between his mathematical formula and the empirical regularity that it represents. By analysing his 1827 mathematical treatise, I will argue that the assumption that tension is equally distributed between every two points in a circuit provided part of the justification for this more general form of coordination.

\footnotetext{
${ }^{9}$ Cf. Tal (2018) for a full exposition of the conventional aspects involved by the construction of measurement scales. It is particularly metric conventions, i.e., the conventions fixing the criteria for the equality of intervals of a quantity, that are crucial for determining the ordering of measurement outcomes and, thus, for achieving measurement coordination between the empirical results of measurement and their representation on a mathematical scale.
} 
The long-standing achievement of Ohm's researches on electrical conductivity is the famous law named after him, which relates intensity of current $(I)$ to voltage or potential difference in modern terms $(V)$, and resistance $(R)$ according to the following equation:

$$
I=V / R
$$

One point on which most historians agree is that Ohm's work on conductivity and resistance introduced important elements of discontinuity in the scientific inquiry on the electric current, especially in the German context. Ohm has been viewed as a key figure anticipating a new wave of research that revolutionised the epistemic standards and values of German electrical science with the use of rigorous mathematics and the search for precise quantification (Caneva 1978). In order to supply a theoretical understanding of his experimental results, Ohm (1827/1891) deployed mathematical tools that were relatively advanced for his time as well as further reasoning tools, including an analogy with Joseph Fourier's theory of heat. ${ }^{10}$ As far as Ohm's theorising is concerned, many historical reconstructions emphasise that a crucial aspect of his work was Ohm's unorthodox use of various notions with respect to how electrical phenomena were conceived in his time (Schagrin 1963, Gupta 1980, Atherton 1986, Archibald 1988, Jungnickel \& McCormmach 2017). More specifically, these authors focus on how Ohm's concept of 'tension' was different from that of Ampère, where the latter was only used with reference to phenomena related to static electricity. Ohm used 'tension' not only to refer to the difference in tension between the endpoints of an open electric circuit, but also to refer to what we would call the voltage of each point of a closed circuit. According to Schagrin (1963), this was the crucial element that allowed Ohm to establish his law, more than any other experimental or material novelty. ${ }^{11}$

In what follows, I provide a reconstruction of Ohm's scientific inquiry on conductivity and resistance in order to analyse the forms of coordination that this involved. To do so, I will use a variety of historical sources, including Ohm's laboratory notes. I will also use the metrological notions of material instruments, physical procedures, instrument readings, measurement outcomes, and calibration, as well as my own distinction between two scales of coordination.

\subsection{Electrical concepts and the measurement of electrical resistance before Ohm}

By the time Ohm began his inquiry into electrical conductivity and resistance in 1825, theorising on static electricity had reached a relatively mature stage, while the inquiry into current phenomena - boosted by Volta's invention of the pile and Ørsted's discovery of electromagnetism - was characterised by discussions on the appropriate concepts to be used for theoretical purposes.

\footnotetext{
${ }^{10}$ Cf. Jungnickel \& McCormmach (2017: 84-94).
}

11 Cf. also Kuhn (1970: 469, footnote 14). 
The $18^{\text {th }}$ century had witnessed the emergence of theories that explained static phenomena - such as electric attraction and repulsion and the charging and discharging of bodies - in terms of the action of one or more 'electrical fluids'. ${ }^{2}$ The understanding of electricity as a fluid substance flowing through solid bodies was therefore a rather entrenched assumption among electrical scientists in the early $19^{\text {th }}$ century. However, by the 1780s, quantities measuring electrical action, that is, the strength of the force producing these static phenomena, could already be defined more precisely. Thanks to a powerful and accurate measurement apparatus based on his torsion studies, Charles Augustin Coulomb managed to empirically establish the inverse law of electrostatics: the reciprocal attraction of negative and positive charges is in the inverse proportion to the square of their distances. ${ }^{13}$ With the concept of 'electrostatic force' he identified the effects resulting from the attraction or repulsion of different opposite charges.

In 1800, Alessandro Volta announced the invention of the pile, which produced stronger and less instantaneous electric effects compared to those known to be produced by static electricity. By alternating zinc and brass strips with cardboard soaked in a salt solution, Volta assembled a battery producing a continuous electric stream that could circulate in an external conductor, what we would refer to as an electric current. To explain the workings of his pile, Volta (1800) distinguished between 'tension', identifying a weak force responsible for static effects, and 'electromotive force', which caused the current to flow and was produced by the amount of electrical fluid set in motion by the contact of different metals.

Volta's distinction introduced crucial differentiation between the new current phenomena and the known static ones. However, electrostatic concepts had a lasting influence in framing the understanding of current phenomena, especially in the context of French science, and the 'electrostatic theory of the pile' remained popular until Hans Christian Ørsted discovered electromagnetism in 1820 (Brown 1969). ${ }^{14}$ Following Ørsted's discovery, André-Marie Ampère introduced a terminological innovation to the study of electric current in order to clarify the distinction between static and current phenomena, thus laying the foundations of the science of electrodynamics. ${ }^{15}$

\footnotetext{
${ }^{12}$ Cf. Heilbron (1979, especially ch. XVIII). In 1734, Charles François de Cisternay du Fay's outlined the two-fluid theory, postulating that electricity was composed of one fluid carrying a positive charge and another one carrying a negative charge. The one-fluid theory, which explained electrical phenomena in terms of one electrical fluid only, was put forward by William Watson in 1746 and by Benjamin Franklin in 1747. The controversy was still alive at the time of Ohm's inquiry.

${ }^{13}$ Gillmor (1972) provides an excellent account of Coulomb's scientific work, while Heilbron (1979: ch. XIX) reconstructs the intricate path towards the emergence of precise quantity concepts in electrostatics (cf. especially pp. 458-473 for earlier attempts at quantifying electrical action and antecedents to Coulomb's law). See below, section 3.2, for more on Coulomb's torsion studies and their relevance for Ohm's experiments and measurement procedure.

${ }^{14}$ Brown discusses how Jean-Baptiste Biot, under the influence of Coulomb's work, initially 'dissolved' Volta's continuous current into a series of discrete shocks and understated the role of contact force between the different metals for the impulsion of electricity.

${ }^{15}$ Cf. Steinle (2016, especially ch. 1-4) for the best account to date of Ørsted's path to the discovery of electromagnetism as well as of Ampère's conceptual innovations. I will discuss Ampère's conceptual refinements and his notion of tension in detail in section 3.4 .
} 
Indeed, experiments on conductivity and resistance had been performed well before the invention of the pile and the observation of current phenomena. ${ }^{16}$ However, the impossibility of experimenting with a continuous current had been a substantial limitation to empirical researches on electrical resistance, since it was impossible to test for its non-instantaneous effects. ${ }^{17}$ By the time Ohm began his inquiry, there was no standard measure of electrical resistance, although several attempts at quantifying its impact on electrical conductivity had led to more precision and reliability in the measuring techniques. ${ }^{18}$ In addition, as we have seen, since theorising over current phenomena was at an early stage, well-defined concepts to investigate current phenomena were still lacking. Ohm, who was trained in both physics and mathematics, chose to focus his scientific efforts on the elucidation of the electric circuit precisely because it was a domain in which much progress - with respect to precise quantification and mathematically-expressed relationships - could still be made (Appleyard 1930). Therefore, he began his experiments with the explicit aim of establishing the law according to which metals conduct current electricity. ${ }^{19}$

\subsection{Ohm's first experimental phase: experiments on 'loss of force' with the battery}

During the first experimental phase, on which he reports in his $(1825 \mathrm{a} / 1892),{ }^{20}$ Ohm attempted to establish how the conductivity of an object is affected by its length. His material apparatus at this stage consisted of a wet cell - a voltaic battery - as the source of the electricity flowing through an external circuit, and a torsion balance to measure the magnetic force exerted by the electric stream when conducting wires of different lengths were inserted into the circuit. ${ }^{21}$

The workings of the torsion balance as an instrument measuring magnetic forces relied on Coulomb's foundational work on the theory of torsion. From the late 1770s, Coulomb began developing a theory of torsion in thin threads, showing how torsion suspension could provide a method to measure small forces with a high level of accuracy (Gillmor 1972: ch. 5). By means of careful experimentation, Coulomb identified a law of torsion, relating the torque (the momentum of the torsion force), a coefficient for the material of the thread, the angle of torsion, and the diameter and length of the thread or wire. Based on this work, he built the first torsion balance, which could be used to measure small magnetic forces. Later in the 1780s, he extended his experimental data to further materials, including metals, and generalised his

\footnotetext{
${ }^{16}$ Already by 1775 , Henry Cavendish had measured through direct sensation the difference in electric charge between glass tubes of different widths filled with a salt solution. Cf. Heilbron (1979: 477-89) for a well-rounded exposition of Cavendish's quantitative concepts and measurement techniques.

${ }^{17}$ Humphry Davy was plausibly the first experimenter to use a steady current and found that wires having the same ratio of length to cross-section had the same resistance.

${ }^{18}$ Cf. Atherton (1986) for a survey of the several different procedures to measure electrical resistance before Ohm.

${ }^{19}$ Cf. Ohm (1825a). According to Schagrin (1963: footnote 18), Ohm used the expression 'contact electricity' to explicitly distinguish his inquiry from researches on static electricity.

20 The paper was published simultaneously in Schweigger's Journal für Chemie und Physik and Poggendorffs Annalen der Physik und Chemie.

${ }^{21} \mathrm{Ohm}$ further experimented with the same set-up to establish the relative conductivity of different metals, on which he reported in his (1825b/1892) and (1826/1892).
} 
law by replacing the coefficient for the material of the thread with a constant rigidity coefficient. Knowledge of torsion laws was, therefore, crucial to building precise torsion balances that could be used for accurate measurements of magnetic and electric phenomena. ${ }^{22}$

In Ohm's setup, the balance consisted of a magnetic needle suspended from a support over one of the conducting wires that made up the circuit connected to the zinc copper battery. When the circuit was closed by connecting the two terminals - the endpoints of the conducting wires - this generated a magnetic interaction between the electricity flowing in the circuit and the suspended magnet which caused a torsion of the needle. When deflected, the needle was brought back to the '0' position by turning the support, and torsion of the suspension was taken as the measure of magnetic force, where 100 parts measured at the micrometre corresponded to a full circle. ${ }^{23}$

In a first series of experiments, Ohm placed wires of different lengths and the same cross-section between the terminals of the circuit, testing for the effects of differences in length against a very short 'standard' wire. While length was his independent variable, the dependent variable being tested was 'loss of force' (Kraftverlust). The quantity term 'loss of force' was operationalised as a reduction in torsion of the magnetic needle while a test wire was in the circuit, compared to that effected when the standard wire was placed into the circuit. ${ }^{24}$ In his 1825 paper, Ohm reports that he systematically alternated measurements taken with the standard wire and measurements with test wires of different lengths. From this first experimental series, which involved several sets of measurements, he obtained the mean values of observed loss of force for each test wire as a percentage of the reduction in force compared to the mean of the values when the standard wire was in place. From these values, he derived an empirical logarithmic equation to describe loss of force, which he then checked empirically by using a very long test wire. He mathematically transformed the equation into the $\log$ arithmic formula $v=m \log (1+x / a)$, where $v$ is the loss of force, $x$ is the length of the conductor, and $a$ and $m$ are empirical constants, the former depending on the internal resistance of the circuit, the latter depending on many factors, including $a$. Subsequently, he reports a second experiment performed after replacing the two wires connected to the battery with others of the same length but a smaller cross-section. From this experiment he reports individual measurements for each test wire to confirm the validity of the equation. After having observed several sources of

\footnotetext{
22 See Gillmor (1972: ch. 6) for more on Coulomb's application of his torsion studies and of his balance to his work on magnetism and electrostatics.

23 According to Heering et al. (2020: 13), this last point may be taken as an indication that the accuracy of the balance was rather poor. In addition, they point out that neither in Ohm's publications nor in his notes is it possible to find exactly which torsion balance he used and the precise distance between the magnetic needle and the conducting wire.

${ }^{24}$ The term that Ohm used to refer to the parameter for resistance seems to have originated with this measurement procedure. In general, by 'reduced length' (reduzierte Länge) Ohm referred to the length of a standard wire, the resistance of which is equivalent to the sum of resistances in the circuit or part of the circuit under investigation (cf. Wheatstone 1843). In this setting, the resistance due to each test wire depended on their respective difference in length compared to the short standard wire and caused a decrease in the intensity of the electric current. The term stuck in his writings until 1829, when he started using the word 'resistance' (Widerstand).
} 
measurement error, particularly due to the behaviour of the battery, he reports having performed a third experiment series by also controlling for the diameter of the rest of the external circuit. From this experiment he reports further individual measurements for each test wire to again confirm the equation.

By comparing Ohm's 1825 paper with his laboratory notes and reproducing his experiments, Heering et al. (2020) draw several interesting conclusions on this experimental phase. Firstly, with respect to the logarithmic form of the equation, they claim that it was not a direct outcome of Ohm's measured data. Rather, it presupposed a conceptual understanding, based on which Ohm calculated adequate numerical results for the empirical constants $a$ and $m$, to be tested against observed measurement outcomes. This claim is supported by the fact that Ohm was selective about the experimental results he chose to publish. Secondly, they highlight that from Ohm's notes we can see that between May 1825, when he performed (among others) the three series of experiments that he reported on in his paper, and July 1825, when he performed further measurements of loss of force, he slightly changed his measurement procedure. I will focus specifically on this latter aspect, clarifying what calibration amounted to during this first experimental phase. This will be crucial to understanding how the change of material and experimental conditions in the second experimental phase - the one that led him to establish his famous law - is related to Ohm's change of measured variable and, thus, measurement coordination.

Ohm's calibration dealt with several aspects of his experimental practice, but he reports only a few of them in his 1825 paper. As mentioned above, Ohm reports that he controlled for the external resistance of the circuit by performing a second series of experiments after replacing the two wires connected to the battery with others of the same length but a smaller cross-section, and a third series after replacing the rest of the external circuit. From the analysis in his notes, Ohm appears to have controlled for the effects of the Earth's magnetic field on the balance needle by reducing the values of the readings taken with the standard wire in the circuit by the values of the readings taken at the end of the whole measurement series. ${ }^{25}$ Finally, Ohm systematically averaged the values of the readings taken with the standard wire, which were taken before and after the measurement with each test wire, because they showed substantial variability. These aspects of calibration are essential to understanding how Ohm accounted for different sources of measurement error to infer measurement outcomes of loss of force from the balance readings. As Heering et al. (2020:17-18) reconstruct from the notes, the values of loss of force were obtained as follows: Ohm divided the difference between each reading of the balance taken when a test wire was in place and the mean of the readings taken when the standard wire was in place by the normal force. The normal force is the reading for the standard wire minus the position of the needle,

\footnotetext{
25 The presence of values for temperature in the notes on the May 1825 experiments also seems to suggest that Ohm presumed a relationship - which he did not investigate systematically - between the outcome of measurement and temperature, possibly due to the internal resistance of the battery, which depends on temperature. Cf. Heering et al. (2020), especially p. 15, footnote 28.
} 
where the latter indicates the torsion produced by the micrometre that is needed to overcome the effect of the Earth's magnetic field.

Ohm's averaging of the balance readings with the standard conductor is an aspect worth focusing on. This form of statistical modelling reduced the source of error due to the variable performance of the wet cell. However, his awareness of the sources of measurement error due to the wet cell seems to have increased between the experiments he performed in May 1825 and those performed in July 1825, leading to some changes in his measurement procedure. A major material difficulty was the polarisation of the wet cell, which was causing sudden surges of electricity when the circuit was opened or closed to replace a test wire. ${ }^{26}$ While in the May 1825 experiments Ohm accounted for this systematic source of measurement error by waiting to observe the instrument reading until the magnetic needle stopped moving, rather than immediately, in the July 1825 experiments Ohm coped with this problem by never opening the circuit to replace the wires he used, but by placing the next wire between the terminals while the previous one was still in place, and then removing the latter. ${ }^{27}$

Another issue was the progressive decrease of the force of the electric stream produced by the wet cell, which Ohm noticed from the decreasing values of the readings taken with the standard wire from the beginning to end of each set of measurements. Since Ohm assumed that the battery performance changed constantly, he averaged the readings with the standard conductor with the clear intention of reducing the impact of this source of measurement error. However, Ohm's increased attention to this phenomenon led him to conduct ad hoc experiments to study the rate of decay of the battery. As McKnight (1967: 111-112) shows, in July 1825, before experimenting on the correlation between loss of force and the length of the conductor, Ohm conducted experiments to study the rate of decay of the battery (fig. 1). Examining Ohm's laboratory notes in the Deutsches Museum Archives, I noticed that these observations were repeated more times, even after the first sets of experiments to relate the length of the conductor to loss of force, as reported in the notes taken in the following days. ${ }^{28}$ Although there is no further evidence of Ohm modelling this source of measurement error, it seems plausible that at this stage Ohm became aware of the importance of methodically understanding whether the battery was constantly decaying, and he may have deemed the average of the values with the standard conductor as insufficient to account for possible systematic measurement error due to a non-stable rate of decay.

\footnotetext{
${ }^{26}$ Polarisation is a mechanical side effect in the battery due to chemical variations in the battery liquid, more specifically, to variations resulting from chemical elements in the electrolyte (i.e., the liquid) being unevenly attracted to the electrodes (i.e., the metals in the battery). Cf. Bagotsky (2006: ch. 6), for a standard contemporary treatment of electrochemical polarisation. ${ }^{27} \mathrm{Ohm}$ reports on this procedural change in his subsequent publication (Ohm 1826: 140-141).

${ }^{28}$ Cf. Item $904-$ NL267/017 of the Ohm Collection, $8^{\text {th }}$ and $9^{\text {th }}$ page.
} 


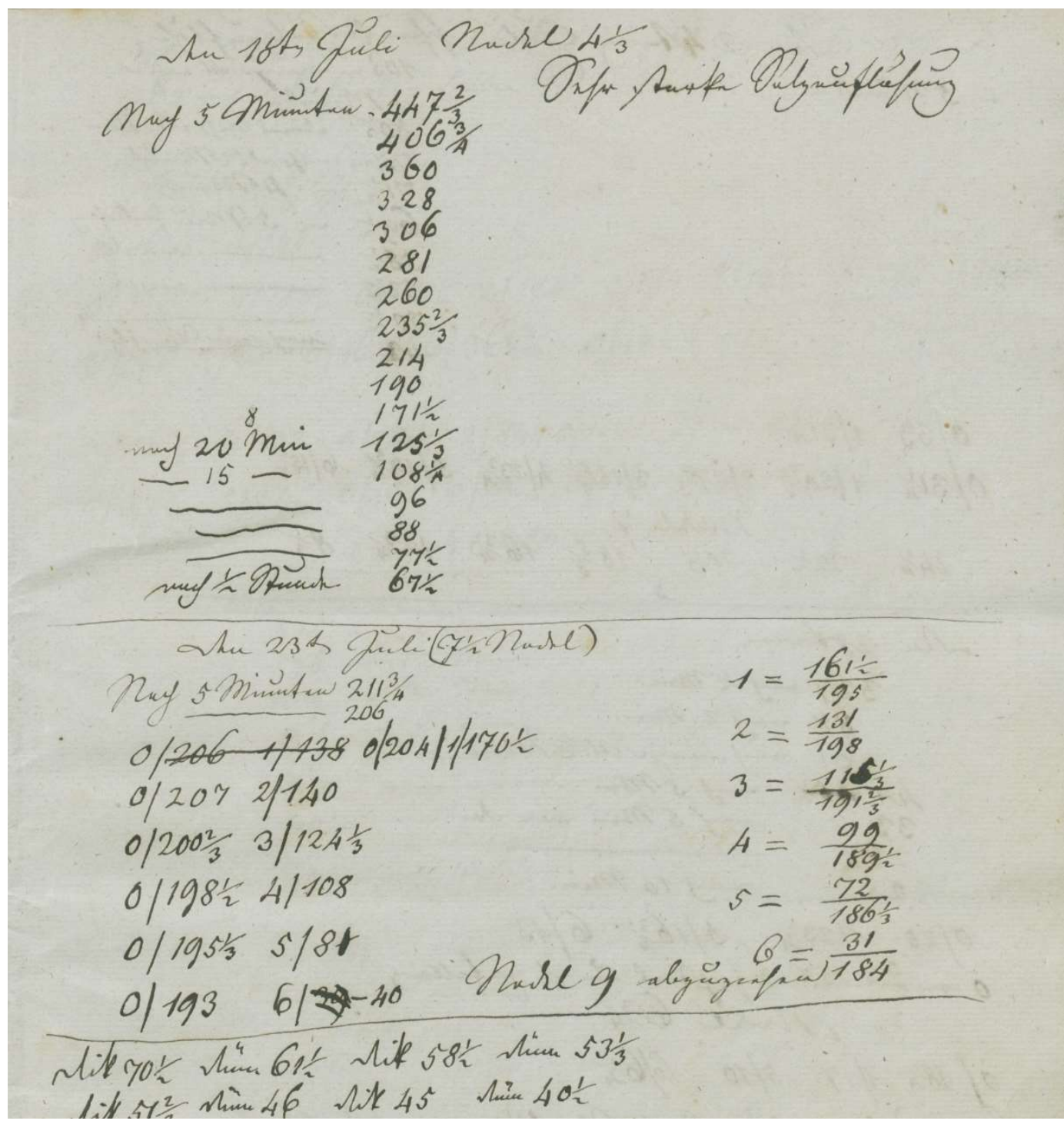

Fig.1: Detail from Ohm's laboratory notebook (18 th and 23 ${ }^{\text {rd }}$ July 1825). The first part of the page shows the experiment on the rate of the decay of the wet cell (note the time indications on the left). The second part shows the actual testing for the effects of resistance. Note the alternation between the standard conductor (indicated by 0) and the test wires (numbered 1 to 6). Item 904 - NL267/017 of the Ohm Collection (7th page). With permission of Deutsches Museum, Munich, Archive, NL_267_017-005.

To sum up, the core elements involved in Ohm's measurement procedure in this first experimental phase are as follows:

- Material instruments: torsion balance (measuring instrument), wet cell (productive instrument).

- Physical procedure: placing different conducting wires into the circuit, noting down the balance readings, bringing back the magnetic needle to the ' 0 ' position.

- Instrument readings: different gradients of deflection of the magnetic needle in the circle of the torsion balance, where 100 parts measured at the micrometre corresponded to a full circle.

- Calibration (with the type of modelling involved indicated in brackets):

1. Modelling the resistance of the external circuit by repeating the experiment with different geometric compositions of the setup (empirical). 
2. Controlling for the effects of the Earth's magnetic field on the balance needle by reducing the values of the readings with the standard wire in the circuit by the values of the readings taken at the end of the whole measurement series (empirical).

3. Avoiding measurement error due to the polarisation of the wet cell by waiting to read the balance readings after replacing the test wire (in the May 1825 experiments), or by keeping the circuit close when replacing the test wires (in the July 1825 experiments) (methodological/pragmatic).

4. Modelling the measurement error due to the rate of decay of the wet cell by averaging the balance readings with the standard conductor (statistical) and by systematically observing the rate of decay of the wet cell (in the July 1825 experiments) (empirical).

- Measurement outcomes: values of loss of force obtained by dividing the difference between each reading of the balance with a test wire and the mean of the readings with the standard wire by the normal force.

- Quantity concept: 'loss of force' (Kraftverlust).

- Theoretical model: $\operatorname{logarithmic}$ formula $v=m \log (1+x / a)$, where $v$ is the loss of force, $x$ is the length of the conductor, and $a$ and $m$ are empirical constants, the former depending on the internal resistance of the circuit, the latter depending on many factors, including $a$.

With respect to the empirical results, historical views mostly converge on taking the logarithmic formula as a good approximation to Ohm's law, especially for short conductors (Schagrin 1963, Heidelberger 1979, Gupta 1980). Indeed, his formula was not reliable beyond a certain length of the conductor, and Ohm himself (1825c/1892: 12) recognised that his approximations failed for very long conductors, that is, when the resistance of the external circuit approached or exceeded the internal resistance of the battery. $^{29}$

Ohm's modelling of the measurement error in this first experimental phase was mostly a matter of careful empirical testing and pragmatic considerations, and a substantial part of it aimed at accounting for the instability of the phenomenon on which he experimented, what we would call an electric current. On the contrary, with respect to his measurement instrument, the torsion balance, calibration only involved controlling for the effects of the Earth's magnetic field. In my discussion above, I have explained that the workings of the balance were underpinned by Coulomb's torsion law and, by the time Ohm started his inquiry, Coulomb's own experimental results provided sufficient justification to trust the reliability of this instrument for the measurement of magnetic and electrostatic phenomena. In addition, Ohm's

\footnotetext{
29 These limitations involve a typical issue within measurement coordination, i.e., the difficulty of extending reliably the structure of a constructed measurement scale beyond the range of application in which it has been calibrated and empirically tested. Cf. Chang (2004: ch. 3) on similar issues with thermometry.
} 
understanding of magnetic effects as a measure of the intensity of electrical force was underpinned by Ampère's force law, which he formulated in 1823 after establishing that the force of attraction or repulsion between two charged wires is proportional to their lengths and to the intensity of their charge.

Since Ohm's measurement procedure relied on the justification provided by Coulomb's torsion law and Ampère's force law, we might wonder what prevented him from measuring the force of the circuit directly, rather than measuring its loss of force due to the resistance of each test conductor compared to that due to minimal resistance of the standard wire. Ohm's choice of 'loss of force' as the variable to test is not surprising, however, since by then it was known that a conductor reduced the magnetic effect of an electric charge inversely to its conductivity; Ohm's choice might also have been influenced by the reasoning of other scientists. ${ }^{30}$ The analysis of his measurement procedure and the assumptions underlying it suggests that the strongest limitations on the reliability of Ohm's measurement procedure came from his productive instrument, the instrument responsible for producing the phenomenon on which to experiment. This suggestion seems corroborated by Ohm's increased attention to the instability of the electricity produced by the wet cell during the July 1825 experiments, when he adopted a more systematic approach to the observation of the rate of decay of the wet cell. In the next section, I show how these aspects are relevant to understanding the change of measurement coordination involved in his change of measured variable in the second experimental phase.

\subsection{Ohm's second experimental phase: experiments on 'exciting force' with the thermocouple}

From December 1825, Ohm began a new series of experiments. He followed the advice of Johann Christian Poggendorff, the editor of the journal in which Ohm had published his previous experimental results, who suggested repeating the experiments by using a copper-bismuth thermocouple instead of a wet cell as a source of electricity (fig. 2). The junction points of this thermoelectric source were immersed in boiling water and melting ice, which were supposed to set a constant temperature differential of 100 ${ }^{\circ} \mathrm{C}$ across the thermocouple and, thus, produce a constant electric stream. The torsion balance was only slightly adapted to fit to the new setting.

\footnotetext{
30 The fact that the poorer the conductor the less the magnetic effect of the electric stream was known at Ohm's time, but none of the many explanations suggested for it had reached a consensus. Schagrin (1963: 541, footnote 21), for instance, refers to J. B. Biot's Précis Éleméntaire de Physique (Deterville, Paris 1821), $2^{\text {nd }}$ ed. as one of Ohm's possible sources. Heering et al. (2020) suggest that Ohm's choice of 'loss of force' as his dependent variable was influenced by Coulomb's third memoir on electricity and magnetism, where he describes mathematically the loss of electrical charge measured with the torsion balance.
} 


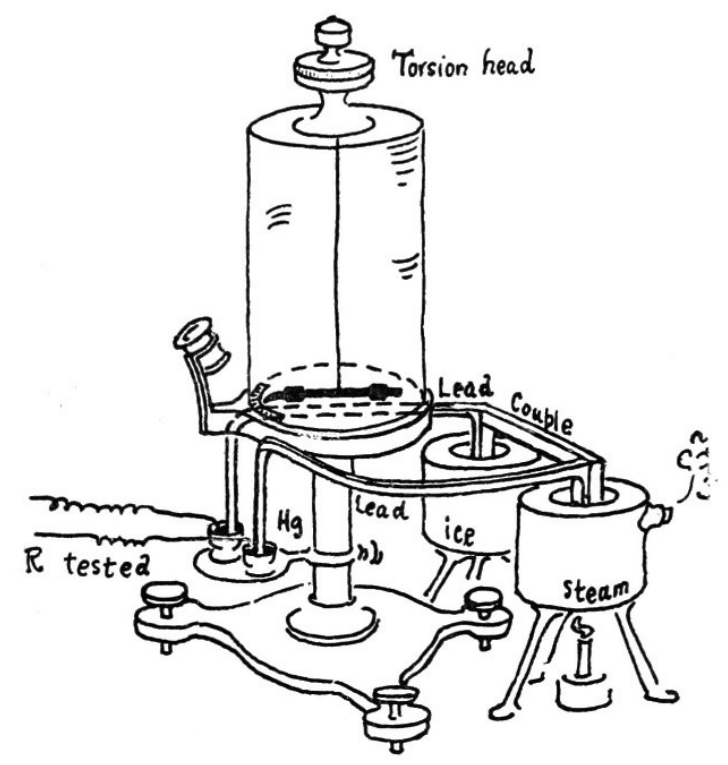

Fig. 2: Ohm's torsion balance and thermocouple. Adapted from "The history of Ohm's law" by J. C., Shedd, \& M. D. Hershey, 1913, Popular Science Monthly, 83, p. 607. CC BY-SA 3.0.

Ohm's laboratory notes present a seemingly smooth process of data gathering. ${ }^{31}$ Three main points stand out in these notes:

1. The electric surges due to the high polarization of the wet cell Ohm observed in the first experimental phase are now entirely absent.

2. The deflection of the needle, measured by the turning of the divided circle, is taken as the measure of the intensity of the electric stream.

3. The standard conductor, crucial in the first set of experiments, is now absent.

This time, Ohm's dependent variable was not 'loss of force'. Instead, he reported the observed force of the electric stream for each test wire; he named this the 'exciting force' (erregenden Kraft) in his 1826 paper, where he reported the results of this second experimental phase. Only later, in his 1827 treatise, did he start using the term 'electromotive force' fitting with the nomenclature introduced by Ampère. This change of the measured variable is crucially related to the fact that Ohm did not use a standard conductor in this second experimental phase. This is because Ohm directly measured the force of the electric stream for each conductor, instead of measuring the loss of force of the electric stream depending on the length of the different conductors compared to a standard conductor. As I will suggest, the concurrent change of the experimental setup, the measurement procedure, and the measured variable from 'loss of force' to 'exciting force' is best understood in terms of a change in the measurement coordination on which Ohm

${ }^{31}$ Cf. Item 904 - NL267/017 of the Ohm Collection, 25 th page. 
relied. However, I should first describe the outcome of Ohm's experiments. The final formula representing his results was:

$$
X=a /(b+x),
$$

where $X$ is the strength of magnetic action (representing current strength), $x$ is the length of the wire, $a$ is the exciting force, and $b$ is the resistance of other parts of the circuit.

Ohm knew the workings of his measuring instrument in this setup, the torsion balance, from the previous experimental phase. In both settings, he used the balance to measure the magnetic force exerted by the electric stream when conducting wires of different lengths were placed in the circuit. The major change in the experimental setup was the thermocouple: the instrument producing the electric stream on which Ohm experimented. In fact, a crucial difference compared to the previous experimental setting was the stability of the electricity produced by the thermocouple. Ohm took the stability of the temperature at the endpoints of the thermocouple as a sign that the intensity of the electric stream was not decreasing across time, which was a major problem with the battery setup. The principle underlying the workings of the copper-bismuth thermocouple was discovered in 1822, when Thomas Seebeck reported on the socalled 'Seebeck effect', that is, the production of a thermomagnetic effect caused by a temperature difference between the ends of two wires of different metals joined at both ends. In 1823, Ørsted put forward an interpretation of the thermomagnetic effects caused by temperature difference in terms of thermoelectric effects. ${ }^{32}$ It was on the basis of this recent and not uncontroversial interpretation that Ohm could consider the thermomagnetic effects produced by the thermocouple - and measured by his torsion balance - as a measure of electric force.

As I have mentioned above, Ohm wrote in his notes that the thermocouple eliminated the violent electric surges when the circuit was opened or closed. However, despite the low internal resistance of the new productive instrument, calibration as empirical modelling could not be entirely dispensed with. In experimental trials conducted in the following days, Ohm attempted to minimise other sources of error and influences of independent variables. ${ }^{33}$ As he did in the previous experimental phase, he controlled for the resistance of the external circuit by changing its composition several times. Ohm also could not obtain his law only by experimenting on an electric stream at a single fixed intensity, i.e. the one set by the $100{ }^{\circ} \mathrm{C}$ temperature differential. To test the robustness of his regularity - in modern terms, to test the invariance of the resistance coefficient independent of current strength - he had to produce electric

\footnotetext{
32 Cf. Ørsted \& Fourier (1823). I thank an anonymous reviewer for stressing this point and for emphasising that this interpretation was not uncontroversial until the late 1820 s.

${ }^{33}$ Cf. Item $904-$ NL267/017 of the Ohm Collection, pages 26 th $39^{\text {th }}$.
} 
streams of different intensities. Here we recall the final formulation of the regularity that he established: $X=a /(b+x)$. By repeating the experiment with different initial temperatures for the thermocouple, Ohm believed that he could determine that the value of $b$ remained the same while that of $a$ changed, $(b+x)$ being the total resistance of the circuit. To test for the effects of the length of the conductors on the exciting force of the circuit, he had to make sure that variations in temperature difference resulted in proportional variations in the magnetic effects measured by the torsion balance. Thus, Ohm had to make sure that magnetic effects, taken as a measure of the intensity of the electric stream, were directly proportional to temperature difference, so that he could properly exclude measurement outcomes of exciting force of the circuit being influenced by variables other than the length of the conductors placed in the circuit. However, in his 1826 paper, Ohm reports carrying out his experiment with only one temperature difference that was not the same as in the basic setting. He immersed one end of the thermocouple in iced water, while the other was left at room temperature "which was shown to be steadily $71 / 2$ degrees Reaumur by a thermometer hanging near the apparatus during the observations" (Ohm 1826: 153$).^{34}$

Below is a list of the elements involved in Ohm's measurement practice in this second experimental phase:

- Material instruments: torsion balance, copper-bismuth thermocouple, thermometer.

- Physical procedures: placing different conductors in the open circuit, noting down the balance readings, bringing the magnetic needle back to the ' 0 ' position.

- Instrument readings: different gradients of deflection of the magnetic needle in the circle of the torsion balance.

- Calibration (with the type of modelling involved indicated in brackets):

1. Modelling the resistance of the external circuit by repeating the experiment with different geometrical compositions of the setup (empirical).

2. Modelling the junction temperature deviations of the thermocouple (empirical).

- Measurement outcomes: values of exciting force obtained without the need for a standard conductor.

- Quantity concept: 'exciting force'.

- Theoretical model: mathematical formula expressing the inverse proportionality between intensity of electricity and resistance, and direct proportionality between the former and exciting force $(X$ $=a /(b+x)$ in Ohm's experimental paper, $I=V / R$ in modern notation, where $R$ results from the aggregation of $x$, the resistance due to the wire's length, and $b$, the resistance of the rest of the circuit).

\footnotetext{
${ }^{34}$ Unfortunately, I have not been able to check how this statement is reflected in Ohm's laboratory notes.
} 
When comparing Ohm's first and second experimental phases, it becomes evident that the change of measured variable from 'loss of force' to 'exciting force' is inextricably intertwined with the change of material and experimental conditions in which Ohm implemented his measurement procedures. Ohm relied on a different measurement coordination in each of the two phases. This means that he relied on a different way to justify how his measurement procedure allowed him to identify the quantity of interest and its possible values. In fact, by comparing the two lists of elements, we see that, in addition to the material apparatus and the measured quantity, the measurement procedure also changed from one phase to the next. More precisely, although the operations concerning the use of test wires and the torsion balance remained quite similar, except for the use of the standard conductor, calibration was very different. These changes in the modelling of the measurement procedure are crucial to understanding how $\mathrm{Ohm}$ relied on a different way of identifying values of the measured quantity, i.e., he relied on different grounds to infer measurement outcomes from the balance readings.

During both experimental stages, Ohm's calibration involved both empirical modelling and reliance on background theoretical commitments. As I showed in section 3.2, in the first experimental phase Ohm inferred measurement outcomes of loss of force based on the complex activity of empirical and statistical modelling of numerous confounding factors and systematic sources of measurement error. This inference also involved the justification provided by Coulomb's law of torsion for the workings of the balance and by Ampère's force law for the interpretation of magnetic effects as a measure of the intensity of electric force.

In the second experimental phase, Ohm's calibration of his measurement procedure was seemingly less burdensome compared to the previous phase, especially with respect to the stability of the current on which he experimented. The available evidence suggests that $\mathrm{Ohm}$ spent limited time modelling confounding factors or systematic sources of measurement error that would alter the stability of the current produced by the thermocouple. The assumption of proportionality between temperature difference and the force of the thermoelectric circuit was a crucial source of justification for his inference of measurement outcomes of exciting force from the balance readings. This assumption was certainly supported by Ørsted's 1823 interpretation of the thermomagnetic effects caused by temperature difference in terms of thermoelectric effects, as well as by Ohm's knowledge that Ampère and Becquerel had shown the direct proportionality of tension and temperature differential between the contact points of an open thermoelectric source. ${ }^{35}$

However, another factor might have contributed to Ohm's relatively limited efforts to calibrate the thermocouple. As I discussed in section 3.2, his awareness of the instability of the electricity produced

\footnotetext{
${ }^{35}$ Cf. Schagrin (1963: 545) and references therein (footnote 37).
} 
by the battery increased in the last part of his first experimental phase, and he had possibly developed further strategies to deal with this, other than the simple averaging of the measurements with the standard wire. ${ }^{36}$ Therefore, Ohm might have been particularly ready to trust the thermocouple as a reliable productive instrument, due to the absence of material complications in the physical procedure, with respect to the stability of the electric stream it produced (Heidelberger 2003). His trust in the material reliability of his productive instrument in (re)producing a phenomenon, i.e. a stable current, was coherent with: the available knowledge of thermo-magnetism; his trust in the accuracy of the available thermometric standards - by which he measured the temperature differences while experimenting; ${ }^{37}$ and the measurement laws involved in his measurement procedure (Ampère's force law and Coulomb's torsion law). Therefore, he felt justified in relying on a different form of measurement coordination to infer values of exciting force from the balance readings.

\subsection{From measurement coordination to general coordination: ‘tension' in Die Galvanische Kette}

When introducing Ohm's inquiry, I pointed out that several historical accounts emphasise that Ohm's use of the notion of 'tension' (Spannung) was discontinuous with that of Ampère, and this conceptual discontinuity has been characterised as a fundamental condition for Ohm's discovery of his law (e.g., Schagrin 1963). In this section, I focus on Ohm's notion of tension, based on the analysis of measurement coordination that I have developed so far. My goal is neither to track the experimental-cognitive pathway leading Ohm to his new concept of tension, nor to argue for the originality of Ohm's notion. ${ }^{38}$ Rather, my aim is to analyse the epistemic role of one of Ohm's assumptions that was central to his understanding of tension. Ohm's assumption that there is the same tension between two adjacent points of an open or closed circuit, although it had an empirical basis, acquired the status of a deductive axiom in his mathematical treatment. What I suggest is that this assumption provided part of the justification for the general coordination between Ohm's mathematical formula and the empirical regularity that it describes, beyond the measurement coordination between exciting force and its measurement procedure that I discussed above.

At the end of the 1780s, Volta started using the term 'tension' to refer to the forces exerted by each point of an electrified body to free itself of its electricity and communicate this to other bodies. ${ }^{39}$ Between 1796 and 1797 , he began suggesting that tension was determined by the "mutual influence of the atmospheres"

\footnotetext{
${ }^{36}$ Cf. Heering et al. (2020), especially p. 23, where they discuss their re-enactment of Ohm's first experiments and suggest that he might have used the 'hissing' of the battery as an acoustic signal to control for when the battery was stable.

37 At the time of Ohm's experiments, controversies on the 'correct' thermometric standard had not yet come to an end (cf. Chang 2004: ch. 2 and 4).

${ }^{38}$ Substantiating these two claims would require a separate treatment and a more in-depth discussion of concept formation in experimental physics. For a recent perspective on concept formation with a historical focus complementary to that of this paper, see Steinle (2016, especially the introduction and ch. 7). For a broader perspective on concept formation in the sciences and their use as investigative tools, see Feest \& Steinle (2012).

${ }^{39}$ Cf. Heilbron (1979: 454) and references therein.
} 
between different conductors; here 'atmosphere' refers to the power of different metals to 'push' the electric fluid (Volta 1918: 475). ${ }^{40}$ As I have mentioned above (section 3.1), after the invention of the pile, Volta distinguished between 'tension' as a weak force causing static effects, and 'electromotive force' produced by the amount of electrical fluid set in motion by the contact of different metals, which caused the current to flow. After Ørsted's discovery of electromagnetism, Ampère (1820) introduced some conceptual refinements to bring more clarity to the distinction between static and current phenomena. With 'electromotive action', he referred to the phenomenon taking place inside the battery cell, which produced an 'electromotive force'. This force caused the electricity to flow within a closed circuit, or it generated tension if the terminals of the circuit were open. ${ }^{41}$ In Ampère's view, tension disappeared at the closing of the circuit because no electrostatic action could be detected. Therefore, the notion of 'tension' only made sense if related to an open circuit (e.g. the endpoints of an open pile), whereas current referred to phenomena taking place in a closed system.

While performing his experiments, Ohm seemingly did not believe that tension vanished once the circuit was closed, as in Ampère's view. With the term 'tension' (Spannung), Ohm referred to the difference in exciting force between two parts of a conductor, which generated the flow of electricity. ${ }^{42} \mathrm{He}$ conceived of the electric flow as a modification in the spatial distribution of exciting force in a conductor, a modification produced by the attempt of the system of conductors to reach an equilibrium state. Therefore, the presence of tension between each pair of points in a conductor was necessary for electricity to keep flowing. This understanding of tension may have initially helped Ohm make sense of his measurement practice, which was based on the continuous 'opening' and 'closing' of the thermoelectric circuit to replace the conducting wires. By assuming that tension was present independent of the circuit being opened or closed, the quantity of exciting force could continue referring to the effects of the electricity flowing in the thermoelectric circuit independent of the presence of a conductor between the endpoints of the circuit. ${ }^{43}$

In Die Galvanische Kette matematisch bearbeitet (1827/1891), Ohm's characterisation of the notion of tension is central to the mathematical derivation of his law. Ohm defines tension as the difference in exciting

\footnotetext{
${ }^{40}$ Cf, also Pancaldi (1990: 128-133).

41 As Caneva (1980: 133, footnote 3) points out, there are many inconsistencies in Ampère's paper with respect to his view on the electric current, but he also points out that Ampère's chief interests in this paper were that of distinguishing between the effects of tension and current electricity and that of developing his theory of magnetism. Brown (1969: 84-85) emphasises that Ampère explicitly refers to Volta's distinction between tension and current effects.

42 Ohm's understanding of tension is comparable to the modern notion of potential differential. However, as pointed out by Maxwell \& Jenkin (1864: 156): “[...] the term [tension] has been somewhat loosely used by various writers, sometimes apparently expressing what we have called the density, and at others diminution of air-pressure. By the most accurate writers it has been used in the sense of a magnitude proportional to potential or difference of potentials [...]."

${ }^{43}$ In some historical accounts (e.g., Archibald 1988), the two terms 'tension' and 'exciting force' are sometimes identified. The translation of Ohm's 1827 treatise itself translates Spannung as 'electroscopic force’ (p. 14, footnote 1). However, this choice obscures the distinct roles of these terms in Ohm's conceptual framework.
} 
force (which in this he calls 'electromotive force') between two parts of a conductor. This difference causes the electricity to flow since the circuit attempts to reach a state of equilibrium. Thus, the constant presence of tension, i.e. of difference in electromotive force, between two parts of the conductor causes the constant transmission of electricity. Ohm developed his mathematical treatment by imagining a homogenous and uniformly thick ring, in which any two adjacent points are characterised by the same tension. ${ }^{44}$ This uniform distribution of tension in the circuit produces the electric flow, since "each particle of the conducting medium situated in the circuit of action receives each moment just the same amount of the transmitted electricity from the one side as it gives off to the other, and therefore constantly retains an unchanged quantity" (Ohm 1827/1891: 22). This means that each point of the electric circuit maintains the same charge, because electricity continuously flows from one point to the one adjacent to it, by virtue of the constant tension between them. In 1825 Becquerel experimentally established that every part of a series circuit has the same electric charge, and this was a fact well known by Ohm, who acknowledges Becquerel's empirical discovery (Ohm 1827/1891: 67). If the electricity flowing through the circuit is the same everywhere (given Becquerel's discovery), and it is only transmitted between adjacent particles, as postulated by $\mathrm{Ohm}$, then tension must be equally distributed along the length of the conductor. To provide a graphic representation (fig. 3), Ohm characterised tension geometrically, by imagining extending the circuit ring to a straight line (line AB). By representing the electromotive force at any point on the circuit as a line departing from that point and perpendicular to the circuit line (lines AF and GB), he could represent tension between any two points in the circuit as the difference in length between any two respective perpendicular lines (line $\mathrm{GH}$ ):

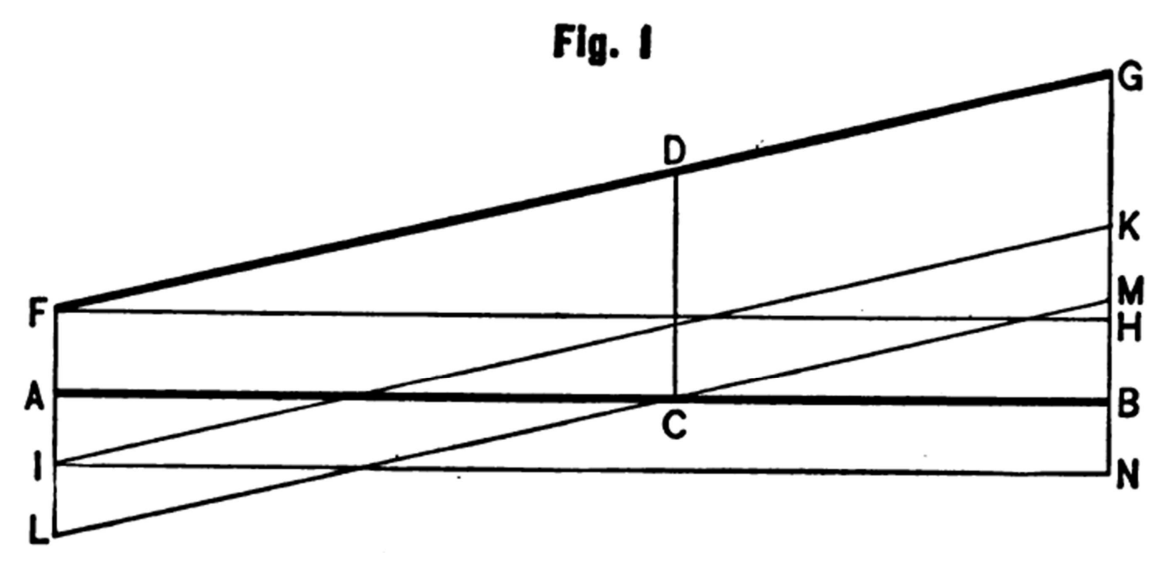

Fig. 3: Ohm's geometric characterisation of tension. Reproduced from Die galvanische Kette, matematisch bearbeitet (p.

23), by G. S. Ohm, 1827. Translated by Francis, W. (1891). The Galvanic Circuit Investigated Mathematically. New York: D. Van Nostrand Company. In the public domain.

\footnotetext{
${ }^{44}$ Further idealising assumptions to model the abstract galvanic circuit were the mono-dimensionality of the propagation of electricity and the assumption that galvanic phenomena do not vary with time, given the constancy in time of the sources of current.
} 
Ohm's assumption that tension is the same between every pair of adjacent points on the circuit was certainly based on Becquerel's empirical discovery that the total electricity is the same in every part of the circuit. However, Ohm $(1827 / 1891: 12)$ himself recognises that there is a hypothetical component to this assumption, when he states that the "law [expressing] the mode of distribution of the electricity within one and the same body $[\ldots]$ is, in part at least, theoretical". The hypothetical component is the assumption that tension is equally distributed between all adjacent points of the circuit, independently of the latter being open or closed. ${ }^{45}$ Even though this assumption was rooted in an empirical fact, Ohm attributed it a definitional status, since he characterised tension as equally distributed in the circuit by defining it as the difference in electromotive force between each pair in an open or closed circuit, as expressed by the geometric characterisation that I described above.

As some commentators have pointed out, by constructing an axiom out of an empirical generalisation, Ohm 'elevated' his assumption to a fundamental principle (Archibald 1988: 145). From an epistemological point of view, this point resonates with Poincaré's (1902) discussion of how certain principles of mechanics are definitions 'in disguise', since they have their origins in experimental regularities, but are elevated to the state of fundamental axioms with an absolute value. As Friedman (2009) suggests, in some cases, the new epistemic status of these principles crucially contributes to the coordination of an empirical regularity with the mathematical formulation that is supposed to represent it. ${ }^{46}$ In my view, this interpretation is helpful to understand what happened with Ohm's notion of tension. Ohm used the assumption of equal distribution of tension, based on Becquerel's inductive generalisation, to justify his definition of 'tension' as the difference in electromotive force between any two points in the circuit. Thus, this theoretical or hypothetical assumption was, on the one hand, based on an empirical generalisation but, on the other hand, Ohm used it in an axiomatic fashion to construct his definition of tension. In this sense, this assumption was providing part of the coordination between Ohm's mathematical formula and the empirical regularity between electromotive force and resistance. This is because, by contributing to the definition of tension, it also justified the possibility of applying that mathematical formula to describe the relationship between electromotive force and resistance in all electric circuits, independent of specific values of electromotive force or of the circuit being open or closed. In sum, the assumption of equal distribution of tension provided part of the general coordination between Ohm's law and the empirical regularity that it describes. However, this assumption was not involved in the measurement coordination between exciting (or electromotive) force and the procedure to measure it. In fact, the latter involved other epistemic components, which were providing the conditions of identification for values of measured quantity. The assumption of equal distribution of

\footnotetext{
${ }^{45}$ Cf., on this point, Schagrin (1963: 546) and Jungnickel \& McCormmach (2017: 88-89).

${ }^{46}$ Friedman makes this point with respect to the role of the principle of relativity and the light principle in Einstein's special theory of relativity.
} 
tension instead provided a condition for applying a general mathematical formula to an empirical regularity.

\section{Conclusion}

In this paper, I have argued for a distinction between two kinds of coordination operating at different scales: measurement coordination and general coordination. Measurement coordination relates to the specific problem of how to justify the attribution of values to a quantity by means of a certain measurement procedure, whilst general coordination refers to the broader issue of justifying the representation of an empirical regularity by means of abstract mathematical tools. To show the fruitfulness of this distinction, I have applied it to the case of Ohm's inquiry on electrical conductivity. Firstly, I have shown how Ohm's measurement procedure and, in particular, his calibration, changed between the first and the second experimental phase. Secondly, I have argued that these differences in calibration determined a different way of justifying the attribution of values to the quantity Ohm measured. Thus, these changes involved a change in measurement coordination between the quantity measured and the procedure to measure it. Therefore, I have suggested that the concurrent change of both Ohm's experimental apparatus and the variable that Ohm measured between the first and second experimental phase should be understood based on the change in measurement coordination on which Ohm relied. Finally, I have shown that Ohm's assumption that tension is equally distributed in the circuit, even if it had an empirical basis, was 'elevated' to the status of an axiom in his mathematical treatise. By contributing to the definition of tension, this assumption justified the possibility of applying Ohm's mathematical formula to describe the relationship between electromotive force and resistance in all electric circuits, independent of specific values of electromotive force or the circuit being open or closed. Therefore, I have argued that the assumption is best understood as a contribution to the justification provided for the general coordination between Ohm's law and the empirical regularity it expresses, rather than to the measurement coordination between exciting force and the procedure for measuring it. In conclusion, the distinction between general and measurement coordination can be a fruitful conceptual tool for the reassessment of several episodes in the history of the sciences. Most importantly, it is a crucial aid to disentangle and analyse the multiple layers of inferential assumptions that enable measurement outcomes to count as evidence in a scientific inquiry, as well as the epistemic dimensions of their justification. 


\section{References}

Ampère, A. M. (1820). Mémoire présenté à l'Académie royale des sciences, le 2 octobre 1820, ou se trouve compris le résumé de ce qui avait été lu à la même Académie les 18 et 25 septembre 1820, sur les effets des courants électriques. Annales de Chimie et de Physique, 15: 59-76.

Appleyard, R. (1930). Pioneers of Electrical Communication. London: MacMillan and Co.

Archibald, T. (1988). Tension and potential from Ohm to Kirchhoff. Centaurus, 31(2): 141-163.

Atherton, T. (1986). A history of Ohm's law. Electronics and Power, 32(6): 467-472.

Bagotsky, V. S. (2006). Fundamentals of Electrochemistry (2nd ed.). Hoboken, NJ: John Wiley \& Sons.

Barwich, A. S., \& Chang, H. (2015). Sensory measurements: coordination and standardization. Biological Theory, 10(3): 200-211.

Boumans, M. (2005). Measurement outside the laboratory. Pbilosophy of Science, 72(5): 850-863.

— (2007). Invariance and calibration. In: Boumans, M., (ed.), Measurement in Economics: A Handbook. Elsevier, 231-248.

Brown, T. M. (1969). The electric current in early nineteenth-century French physics. Historical Studies in the Physical Sciences, 1: 61-103.

Campbell, N. R. (1920). Physics: The Elements. London: Cambridge University Press.

Caneva, K. L. (1978). From Galvanism to electrodynamics: the transformation of German physics and its social context. Historical Studies in the Physical Sciences, 9: 63-159.

- (1980). Ampère, the etherians, and the Oersted connexion. The British Journal for the History of Science, 13(2): 121-138.

Cartwright, N., \& Bradburn, N. (2011) A theory of measurement. In: The importance of common metrics for advancing social science theory and research: a workshop summary. Washington: National Academies Press, 53-56.

Chang, H. (1995). Circularity and Reliability in Measurement. Perspectives on Science 3(2): 153-172

. (2004). Inventing Temperature: Measurement and Scientific Progress. Oxford: Oxford University Press.

Feest, U., \& Steinle, F., (Eds.), (2012). Scientific Concepts and Investigative Practice. Berlin: De Gruyter.

Friedman, M. (2001). Dynamics of Reason. Stanford: Csli Publications.

(2009). Einstein, Kant, and the relativized a priori. In: Bitbol, M., Kerszberg, P. and Petitot, J., (Eds.), Constituting Objectivity: Transcendental Perspectives on Modern Physics. Netherlands: Springer, 253-267.

Frigerio, A., Giordani, A., \& Mari, L. (2010). Outline of a general model of measurement. Synthese, 175(2): 123-149.

Gillmor, C. S. (1972). Coulomb and the Evolution of Physics and Engineering in Eighteenth-Century France. Princeton University Press

Giordani, A., \& Mari, L. (2012). Measurement, models, and uncertainty. IEEE Transactions on Instrumentation and Measurement, 61(8): 2144-2152. 
Gupta, M. S. (1980). Georg Simon Ohm and Ohm's Law. IEEE Transactions on Education, 23(3): 156-162.

Heering, P., Keck, J., \& Rohlfs, G. A. (2020). Laboratory Notes, Laboratory Experiences, and Conceptual Analysis: Understanding the Making of Ohm's First Law in Electricity. Berichte zur Wissenschaftsgeschichte, 43(1): 7-27.

Heidelberger, M. (1979). Der Wandel der Elektrizitätslehre ₹u Ohms Zeit (PhD thesis, LMU München).

- (1980). Towards a logical reconstruction of revolutionary change: the case of Ohm as an example. Studies in History and Philosopby of Science Part A, 11(2): 103-121.

— (2003). Theory-ladenness and scientific instruments in experimentation. In: Radder, H., (ed.), The Pbilosopby of Scientific Experimentation. Pittsburgh, PA: University of Pittsburgh Press, 138-151.

Heilbron, J. L. (1979). Electricity in the 17th and $18^{\text {th }}$ Century. A Study of Early Modern Physics. University of California Press

Johansson, I. (2014). Constancy and circularity in the SI. Metrology Bytes. www.metrologybytes.net/PapersUnpub/OpEds/Johansson_2014.pdf. (accessed 2 February 2018).

Jungnickel, C., \& McCormmach, R. (2017). The Second Physicist. Berlin: Springer.

Kuhn, T. S. (1970). Second thoughts on paradigms. In: Suppe, F., (ed.), The Structure of Scientific Theories. Urbana: University of Illinois Press, 459-482.

Mach, E. (1896). Principles of the Theory of Heat. T. J. McCormack (trans.), (1986), Dordrecht: D. Reidel.

Mari, L. (2003). Epistemology of measurement. Measurement, 34(1): 17-30.

Maxwell, J. C., \& Jenkin, F. (1864). Appendix C.-On the elementary relations between electrical measurements. In: Report of the thirty-third meeting of the British Association for the Advancement of Science; held at Newcastle-upon-Tyne in August and September 1863, 130-163. London: John Murray.

McClimans, L. (2013). The role of measurement in establishing evidence. Journal of Medicine and Philosophy, 38(5): 520-538.

McClimans, L., Browne, J., \& Cano, S. (2017). Clinical outcome measurement: Models, theory, psychometrics and practice. Studies in History and Philosopby of Science Part A, 65: 67-73.

McKnight, J. L. (1967). Laboratory notebooks of GS Ohm: a case study in experimental method. American Journal of Physics, 35(2): 110-114.

Michel, M. (2019). The mismeasure of consciousness: A problem of coordination for the Perceptual Awareness Scale. Philosophy of Science, 86(5): 1239-1249.

Ørsted, H. C., \& Fourier, J. B. (1823). Sur quelques nouvelles expériences thermoélectriques faites par M. le Baron Fourier et M. Oersted. Annales de Chimie et de Physique, 22: 375-389.

Ohm, G. S. (1825a). Vorläufige Anzeige des Gesetzes, nach welchem Metalle die Contact-Elektricität leiten. Schweigger's Journal für Chemie und Physik, Bd. 44, 110-118, and Poggendorff's Annalen der Physik und Chemie, Bd. 4, 79-88. Reprinted in: Lommel, E., (ed.), (1892). Gesammelte Abhandlungen von G.S Ohm. Leipzig: Arthur Meiner, 1-8.

(1825b). Ueber Leitungsfähigkeit der Metalle für Elektricität. Scbweigger's Journal für Chemie und Physik, Bd. 44, 245-247. Reprinted in: Lommel, E., (ed.), (1892). Gesammelte Abhandlungen von G.S Ohm. Leipzig: Arthur Meiner, 9-10. 
- (1825c). Ueber Elektricitätsleiter. Schweigger's Journal für Chemie und Physik, Bd. 44, 370-373. Reprinted in: Lommel, E., (ed.), (1892). Gesammelte Abhandlungen von G.S Ohm. Leipzig: Arthur Meiner, 11-13.

- (1826). Bestimmung des Gesetzes, nach welchem Metalle die Contactelektricität leiten, nebst einem Entwurfe zu einer Theorie des Voltaischen Apparates und des Schweiggerschen Multiplicators. Schweigger's Journal 'für Chemie und Physik, Bd. 46, 137-166. Reprinted in: Lommel, E., (ed.), (1892). Gesammelte Abhandlungen von G.S Ohm. Leipzig: Arthur Meiner, 14-36.

- (1827). Die galvanische Kette, matematisch bearbeitet. Translated by Francis, W. (1891). The Galvanic Circuit Investigated Mathematically. New York: D. Van Nostrand Company.

Padovani, F. (2015). Measurement, coordination, and the relativized a priori. Studies in History and Philosophy of Science Part B: Studies in History and Philosopby of Modern Physics, 52: 123-128.

- (2017). Coordination and measurement: What we get wrong about what Reichenbach got right. In: Massimi, M., Romeijn, J. W., \& Schurz, G., (Eds.), EPSA15 Selected Papers. European Studies in Pbilosophy of Science, 5, 49-60.

Pancaldi, G. (1990). Electricity and life. Volta's path to the battery. Historical Studies in the Physical and Biological Sciences, 21(1): 123-160.

Poincaré, H. (1902). La Science et l'Hypothèse. English translation in: Science and Hypothesis. New York: Dover Books, 1952.

Reichenbach, H. (1920). Relativitätstheorie und Erkenntnis Apriori. Berlin: Springer. English translation in: The Theory of Relativity and A Priori Knowledge. Berkeley/Los Angeles, CA: University of California Press, 1965.

Riordan, S. (2015). The objectivity of scientific measures. Studies in History and Pbilosophy of Science Part A, 50: 38-47.

Ruthenberg, K., \& Chang, H. (2017). Acidity: Modes of characterization and quantification. Studies in History and Philosopby of Science Part A, 65: 121-131.

Schagrin, M. L. (1963). Resistance to Ohm's law. American Journal of Physics, 31(7): 536-547.

Shedd, J. C., \& Hershey, M. D. (1913). The history of Ohm's law. Popular Science Monthly, 83: 599-614.

Sherry, D. (2011). Thermoscopes, thermometers, and the foundations of measurement. Studies in History and Philosophy of Science Part A, 42(4): 509-524.

Steinle, F. (2016). Exploratory Experiments. Amperre, Faraday, and the Origins of Electrodynamics. University of Pittsburgh Press.

Stevens, S.S. (1946). On the theory of scales of measurement. Science, 103: 677-680.

Suppes, P. (1951). A set of independent axioms for extensive quantities. Portugaliae Mathematica, 10: 163-172.

Tal, E. (2013). Old and new problems in philosophy of measurement. Philosophy Compass, 8(12): 1159-1173.

(2016a). Making time: A study in the epistemology of measurement. The British Journal for the Philosophy of Science, 67(1): 297-335.

- (2016b). How Does Measuring Generate Evidence? The Problem of Observational Grounding. In Journal of Physics: Conference Series (Vol. 772, No. 1, p. 012001). IOP Publishing. 
— (2017a). Measurement in Science. In: Zalta, E. N.: The Stanford Encyclopedia of Pbilosophy (Fall 2017 Edition), $\mathrm{URL}=<$ https://plato.stanford.edu/archives/fall2017/entries/measurement-science/ $>$.

—_ (2017b). A model-based epistemology of measurement. In: Mößner, N., and Nordmann, N., (Eds.), Reasoning in Measurement. London and New York: Routledge, 233-253.

- (2017c). Calibration: Modelling the measurement process. Studies in History and Pbilosophy of Science Part A, 65: $33-45$.

- (2018). Naturalness and convention in the International System of Units 败. Measurement, 116: 631-643.

— (2019). Individuating quantities. Philosophical Studies, 176 (4): 853-878.

van Fraassen, B. C. (2008). Scientific Representation: Paradoxes of Perspective. New York: Oxford University Press.

Volta, A. (1800). On the electricity excited by the mere contact of conducting substances of different kinds, Philosophical Transactions, 90: 403-431.

— (1918). Opere. Milano: Hoepli.

Wheatstone, C. (1843). XIII. The Bakerian lecture. An account of several new instruments and processes for determining the constants of a voltaic circuit. Philosophical Transactions of the Royal Society of London, 133: 303327.

Winter, H. J. J. (1944). The reception of Ohm's electrical researches by his contemporaries. The London, Edinburgh, and Dublin Pbilosophical Magazine and Journal of Science, 35(245): 37. 